\title{
Economic evaluations of guideline-based care for chronic wounds: a systematic review
}

\author{
Qinglu Cheng ${ }^{1 *}$, Nicholas Graves ${ }^{1}$, Rosana E. Pacella ${ }^{1,2}$ \\ ${ }^{1}$ Australian Centre for Health Services Innovation (AusHSI), Institute of Health and Biomedical \\ Innovation, Queensland University of Technology, Brisbane, QLD, Australia \\ ${ }^{2}$ Wound Management Innovation Cooperative Research Centre, Brisbane, QLD, Australia
}

\section{*Corresponding Author}

E-mail: qinglu.cheng@hdr.qut.edu.au

Telephone: +61410211108

Running heading: Economic evaluations of guideline-based care for chronic wounds 


\section{ABSTRACT \\ Objectives}

The aim of this study was to review all published economic evaluations of guideline-based care for chronic wounds and to assess how useful these studies are for decision making in health services.

\section{Methods}

Embase, PubMed, Scopus, Health Technology Assessment (HTA) and National Health Service Economic Evaluation Database (NHS EED) were searched on April 16 ${ }^{\text {th }}, 2018$. We included studies that evaluated the economic impact and health outcomes associated with implementing evidencebased guidelines as a bundle of care for the prevention and/or treatment of chronic wounds. Information was extracted from each eligible study and organised by the type of chronic wound. The quality of published economic evaluation studies was assessed using the Consolidated Health Economic Evaluation Reporting Standards (CHEERS).

\section{Results}

A total of 24 economic evaluation studies met the inclusion criteria, of which 12 applied decision analytic models. The compliance with the CHEERS checklist ranged between $43 \%$ and $83 \%$.

\section{Limitations}

We may have missed some economic evaluation studies despite the use of broad search terms. The quality assessment was conducted based on judgment. Using the CHEERS checklist may reflect the way evaluations were reported rather than conducted.

\section{Conclusions and implications of key findings}

We found that guideline-based care may be cost-saving or cost-effective in most circumstances. The quality and usefulness of reviewed studies for decision making was variable. Better information and higher-quality economic evaluations will increase decision makers' confidence to promote guidelinebased care.

\section{Systematic review registration number}

PROSPERO CRD42017051859 


\section{Key Points for Decision Makers}

Providing better wound management based on guidelines may improve patient outcomes at a lower cost or at some acceptable additional cost.

There are variations in the quality of reviewed economic evaluation studies, which may limit the usefulness of those studies to decision making. 


\section{INTRODUCTION}

Chronic wounds are skin lesions that take more than 3 months to heal [1]. The most common chronic wounds seen in general practice include diabetic foot ulcers (DFUs), venous leg ulcers (VLUs), pressure ulcers (PUs) and arterial ulcers (AUs) [2]. Chronic wounds have been described as a silent epidemic as they are often disguised as comorbid conditions [3]. For example, DFU is usually defined as a complication of diabetes. In fact, chronic wounds are affecting a large proportion of the world's population. In the UK, it is estimated that 70,000-190,000 individuals have VLUs at any time and around 400,000 individuals develop a new PU annually[4]. In the USA, more than 6 million people are affected by chronic wounds [3]. The incidence and prevalence of chronic wounds will continue to rise, fuelled by the aging population and increasing numbers of diseases and conditions such as diabetes and obesity. Chronic wounds not only reduce the quality of life of affected individuals [5-7], but also are associated with substantial costs. In Australia, the total cost of chronic wounds was estimated at USD $\$ 2.85$ billion annually [8], but this estimate was highly uncertain due to lack of Australian data on incidence of chronic wounds. In the UK, the cost of managing chronic wounds was conservatively estimated at $£ 2.3-3.1$ billion per year [4]. A recent study in the US estimated that total Medicare spending in 2014 for all wound types ranged from $\$ 28.1$ to $\$ 96.8$ billion [9].

Clinical guidelines have been developed across the globe incorporating best available evidence to manage chronic wounds. The International Working Group on the Diabetic Foot has been publishing and updating guidance documents on prevention and management of foot problems in diabetes since 1999 [10]. The Australia and New Zealand clinical practice guideline for prevention and management of venous leg ulcers [11] summarizes best information available on the assessment, diagnosis, management and prevention of VLUs within the Australian and New Zealand health care context. The National Pressure Ulcer Advisory Panel (NPUAP), European Pressure Ulcer Advisory Panel (EPUAP) and Pan Pacific Pressure Injury Alliance (PPPIA) have collaborated and developed an international guideline on the prevention and treatment of PUs [12]. Apart from the three guidelines mentioned, there are many other guidelines from different organizations and countries. However, there is often a gap between guidelines and implementation, which can be caused by lack of reimbursement and incentives and poor education and training among health professionals [13]. Where wound care followed clinical evidence, studies have found that healing rates were improved and recurrence reduced $[14,15]$. However, implementing guideline-based care usually incurs additional costs to the health system and patients, and little is known about whether the investment in guideline-based care can be offset or outweighed by improved healing, fewer complications or fewer wound recurrences.

Carter [16] conducted a systematic review on economic evaluations of guideline-based or strategic Interventions for the prevention or treatment of chronic wounds. However, some studies included in Carter's review evaluated strategic interventions not relevant to published guidelines. For example, Carter's review included a study by Gordon et al. [17] where authors examined the costeffectiveness of a community Leg $\mathrm{Club}^{\circledR}$ model for chronic VLU management compared with traditional community home nursing. The study, however, did not compare guideline-based care to standard care because participants in both intervention and control groups received comprehensive assessment and compression therapy. The main difference between comparators lay in the location of wound care rather than whether guideline-based care was implemented. Carter also limited eligibility to studies that included at least one incremental cost-effectiveness ratio (ICER) or incremental net health benefit (INHB) or provided costs and benefits so that ICERS/INHBs could be 
calculated. But this would exclude economic evaluations conducted earlier. For example, Thomson et al. [18] published a pilot economic evaluation of a potential PU prevention programme in 1999 but reported costs and benefits with a range rather than mean or median value. Thus, an explicit ICER or INHB could not be derived from the study. But the study still provided some evidence that the benefits outweighed the costs and should be considered for its usefulness to decision making.

The aim of this study was to conduct a systematic review of published economic evaluations of guideline-based wound care that incorporates clinical evidence to treat and/or prevent chronic wounds in the adult population and to assess how useful these studies are in providing information for decision makers in this field. We also aimed to address some limitations presented in Carter's review in our systematic review in addition to including more recently published studies.

\section{METHODS}

This systematic review followed the Preferred Reporting Items for Systematic Reviews and MetaAnalyses (PRISMA) Statement [19]. Searches were conducted in the following databases on April $16^{\text {th }}$, 2018: Embase, PubMed, Scopus, Health Technology Assessment (HTA) and National Health Service Economic Evaluation Database (NHS EED). The systematic review protocol was published in PROSPERO (ID: CRD42017051859). Search terms used in each database were summarized in the Appendix.

Studies were eligible for inclusion if they: were economic evaluation studies (cost-effectiveness, cost-utility or cost-benefit); evaluated the economic impact and health outcomes associated with implementing guideline-based recommendations (a bundle of care) for the prevention and/or treatment of chronic wounds. Studies were excluded if they: evaluated a single product or intervention for the treatment or prevention of chronic wounds; were published as conference abstract, letters, and editorials, note and short surveys; or were cost analyses where health outcomes were not evaluated. The search was not restricted to the English language nor by publication period. Full inclusion and exclusion criteria for selection of studies to be included in this review are given in the protocol published in PROSPERO (ID: CRD42017051859).

Initial selection of studies was based on screening titles and abstracts. Full articles of potentially relevant studies were assessed for eligibility. Final decisions regarding the inclusion or exclusion of studies were made on a consensus between both reviewers (REP and QC). Reference lists of included studies were scanned for other potential studies. Key information from each included study was extracted into a data table.

The quality of published economic evaluation studies was assessed using the Consolidated Health Economic Evaluation Reporting Standards (CHEERS) [20]. The 24-item checklist is a consolidation and update of previous reporting guidelines. It consists of recommendations on reporting methods and findings for economic evaluation studies and provides a means of comparing studies. We used the CHEERS checklist not only because it has been endorsed by several journals as a guideline to report methods and results, but also it helps determine the usefulness of those studies to decision/policy makers. A simple conclusion whether a practice is cost-effective or not will not suffice as a support to change policies. Information on the target population, what clinical effectiveness the study relies on and how uncertainty impacts on the results is also required to complete the message we want to convey to decision/policy makers. When scoring the included studies against the CHEERS checklist, we used 1 for an item that met the criteria, 0.5 for item that partially met the criteria and 0 when the study did not meet the criteria. We also assessed overall compliance with the CHEERS checklist by calculating the proportion of the CHEERS criteria addressed by the study. Fully meeting the 
criteria would contribute 1 to the numerator while partially meeting the criteria would contribute 0.5 to the numerator. Any criteria items that were not applicable to the study were excluded from the denominator.

\section{RESULTS}

A total of 5628 records were identified through the search and additional sources. After duplicate removal and initial screening, 51 full articles were assessed for eligibility and 24 met the inclusion criteria $[18,21-43]$. The reasons for exclusion are shown in Figure 1.

Figure 1. PRISMA flow diagram showing process of study selection for inclusion in systematic review

\subsection{Overview of included studies}

Tables $1-4$ summarize the data extraction from each study. Of the 24 included studies that evaluated the costs and health outcomes of implementing guideline-based practice for chronic wounds, 7 focused on DFU [21-27], 8 on PUs [18, 28-34] , 4 on VLUs [35-38] and 5 on mixed types of chronic wounds [39-43]. Ten studies referred to evidence-based or specialist wound care or gold standard care but did not explicitly mention whether the intervention was guideline-based $[18,28$, $29,35,36,38-41,43]$. We still included these studies in this review, but adjusted the quality score accordingly.

Economic evaluations were conducted either 1 ) alongside randomized controlled trials (RCTs) or using other study designs (referred to as "not model-based" in this paper) or 2) use economic modelling techniques such as decision trees and Markov models ("model-based" studies). Twelve out of the 24 included studies were model-based: ten studies developed Markov models [22-25, 27$29,31,36,40]$ and 2 studies used decision tree models [21, 30] for the analysis, but a figure illustrating the model structure was not provided in 2 studies [25, 29]. The remaining 12 studies compared the implementation of guideline-based care with non-implementation through prospective cohort study and retrospective data audit $[26,38,39,41]$, prospective cohort study and retrospective data audit [33,37], quasi-experimental pre/post study [34, 35, 42], cluster randomized trial $[32,43]$ or a prevalence and incidence study [18].

Outcome measures used in these 24 studies varied by study design. All 12 studies that applied models reported outcomes using incremental cost-effectiveness ratios (ICER) which were calculated by dividing the difference in cost between two practices by the difference in their effectiveness. Other studies reported change in costs and clinical outcomes either separately, or in a specific form, such as cost per ulcer-free day. Most studies demonstrated that implementing guideline-based care would be cost-saving or cost-effective except for 2 studies. Whitty et al. [32] applied costeffectiveness and cost-benefit analysis to evaluate a pressure prevention bundle. They found that net monetary benefit for the intervention was negative. Graves et al. [40] constructed a Markov model and compared total costs and health outcomes before and after the patients' admission to a specialist wound clinic. They found that specialist wound care reduced costs but there was a very slight decrement in QALYs for patients receiving evidence-based wound care. 
Table 1. Summary characteristics of economic evaluation studies for DFU

\begin{tabular}{|c|c|c|c|c|c|c|c|c|c|c|c|}
\hline Author (Setting) & $\begin{array}{l}\text { Publication } \\
\text { Year }\end{array}$ & Type of care & Intervention & Comparator & Analysis & Study design & Perspective & $\begin{array}{l}\text { Time } \\
\text { horizon/ } \\
\text { Study } \\
\text { period }\end{array}$ & $\begin{array}{l}\text { Outcome } \\
\text { measures }\end{array}$ & $\begin{array}{l}\text { Baseline } \\
\text { analysis }\end{array}$ & $\begin{array}{l}\text { Sensitivity } \\
\text { analysis }\end{array}$ \\
\hline $\begin{array}{l}\text { Cardenas et al. } \\
\text { [21] } \\
\text { (Peru) }\end{array}$ & 2015 & Prevention & $\begin{array}{l}\text { Standard care based on } \\
\text { International Diabetes } \\
\text { Federation guidelines }\end{array}$ & $\begin{array}{l}\text { Sub-optimal } \\
\text { care }\end{array}$ & CEA & Decision tree & Societal & 1 year & $\begin{array}{l}\text { Cost per deaths } \\
\text { averted }\end{array}$ & Cost-saving & Robust (OW) \\
\hline $\begin{array}{l}\text { Cheng et al.[22] } \\
\text { (Australia) }\end{array}$ & 2016 & $\begin{array}{l}\text { Prevention } \\
\text { and treatment }\end{array}$ & $\begin{array}{l}\text { Optimal care based on } \\
\text { National Evidence } \\
\text { Based Guidelines on } \\
\text { Prevention, } \\
\text { Identification and } \\
\text { Management of Foot } \\
\text { Complications in } \\
\text { Diabetes }\end{array}$ & Usual care & CUA & $\begin{array}{l}\text { Markov model ( } 7 \\
\text { health states) }\end{array}$ & Health system & 5 years & $\Delta \operatorname{cost} / \triangle Q A L Y$ & Cost-saving & $\begin{array}{l}\text { Robust (SA, } \\
\text { PSA) }\end{array}$ \\
\hline $\begin{array}{l}\text { Ortegon et al. } \\
\text { [23] } \\
\text { (Netherlands) }\end{array}$ & 2004 & $\begin{array}{l}\text { Prevention } \\
\text { and treatment }\end{array}$ & $\begin{array}{l}\text { Optimal prevention } \\
\text { and treatment } \\
\text { according to } \\
\text { International } \\
\text { consensus on diabetic } \\
\text { foot }\end{array}$ & $\begin{array}{l}\text { Present level of } \\
\text { prevention }\end{array}$ & CUA & $\begin{array}{l}\text { Markov model } \\
\text { (13 health states) }\end{array}$ & $\begin{array}{l}\text { Not reported } \\
\text { (health system) }\end{array}$ & Life time & $\Delta \operatorname{cost} / \triangle Q A L Y$ & $\begin{array}{l}\text { Cost- } \\
\text { effective }\end{array}$ & Robust (OW) \\
\hline $\begin{array}{l}\text { Ragnarson } \\
\text { Tennvall et al. } \\
\text { [24] } \\
\text { (Sweden) }\end{array}$ & 2001 & Prevention & $\begin{array}{l}\text { Optimal patient } \\
\text { prevention according } \\
\text { to International } \\
\text { consensus on diabetic } \\
\text { foot }\end{array}$ & $\begin{array}{l}\text { Present level of } \\
\text { prevention }\end{array}$ & CUA & $\begin{array}{l}\text { Markov model ( } 8 \\
\text { health states) }\end{array}$ & $\begin{array}{l}\text { Not reported } \\
\text { (health system) }\end{array}$ & 5 years & $\Delta \operatorname{cost} / \triangle Q A L Y$ & $\begin{array}{l}\text { Not cost- } \\
\text { effective } \\
\text { for low risk } \\
\text { group; } \\
\text { cost-saving } \\
\text { or cost- } \\
\text { effective } \\
\text { for other } \\
\text { risk groups }\end{array}$ & Robust (OW) \\
\hline $\begin{array}{l}\text { Rauner et al. [25] } \\
\text { (Austria) }\end{array}$ & 2005 & Prevention & $\begin{array}{l}\text { Intensified prevention } \\
\text { program based on } \\
\text { International } \\
\text { consensus on diabetic } \\
\text { foot }\end{array}$ & $\begin{array}{l}\text { Present } \\
\text { prevention }\end{array}$ & CUA & $\begin{array}{l}\text { Markov model ( } 8 \\
\text { health states) }\end{array}$ & $\begin{array}{l}\text { Not reported } \\
\text { (health system) }\end{array}$ & 10 years & $\Delta \operatorname{cost} / \triangle Q A L Y$ & $\begin{array}{l}\text { Not cost- } \\
\text { effective } \\
\text { for low risk } \\
\text { group; } \\
\text { cost-saving } \\
\text { or cost- } \\
\text { effective }\end{array}$ & Robust (OW) \\
\hline
\end{tabular}




\begin{tabular}{|c|c|c|c|c|c|c|c|c|c|c|c|}
\hline & & & & & & & & & & $\begin{array}{l}\text { for other } \\
\text { risk groups }\end{array}$ & \\
\hline $\begin{array}{l}\text { Rerkasem et al. } \\
\text { [26] } \\
\text { (Thailand) }\end{array}$ & 2009 & $\begin{array}{l}\text { Prevention } \\
\text { and treatment }\end{array}$ & $\begin{array}{l}\text { Multidisciplinary } \\
\text { Diabetic Foot Protocol }\end{array}$ & Standard care & CEA & $\begin{array}{l}\text { Retrospective } \\
\text { data audit }\end{array}$ & $\begin{array}{l}\text { Not reported } \\
\text { (societal) }\end{array}$ & $\begin{array}{l}3 \text { years } 7 \\
\text { months* }\end{array}$ & $\begin{array}{l}\text { Costs and SF-36 } \\
\text { score }\end{array}$ & Cost-saving & NA \\
\hline $\begin{array}{l}\text { Wu et al. [27] } \\
\text { (China) }\end{array}$ & 2018 & Prevention & $\begin{array}{l}\text { Prevention based on } \\
\text { the recommendations } \\
\text { of experts and IWGDF } \\
\text { guidance documents }\end{array}$ & Usual care & CUA & $\begin{array}{l}\text { Markov model ( } 8 \\
\text { health states) }\end{array}$ & Healthcare & Life time & $\Delta \operatorname{cost} / \triangle Q A L Y$ & Cost-saving & $\begin{array}{l}\text { Robust (OW, } \\
\text { PSA) }\end{array}$ \\
\hline
\end{tabular}


Table 2. Summary characteristics of economic evaluation studies for PU

\begin{tabular}{|c|c|c|c|c|c|c|c|c|c|c|c|}
\hline $\begin{array}{l}\text { Author } \\
\text { (Setting) }\end{array}$ & $\begin{array}{l}\text { Publication } \\
\text { Year }\end{array}$ & Type of care & Intervention & Comparator & Analysis & Study design & Perspective & $\begin{array}{l}\text { Time horizon/ } \\
\text { Study period }\end{array}$ & $\begin{array}{l}\text { Outcome } \\
\text { measures }\end{array}$ & $\begin{array}{l}\text { Baseline } \\
\text { analysis }\end{array}$ & $\begin{array}{l}\text { Sensitivity } \\
\text { analysis }\end{array}$ \\
\hline $\begin{array}{l}\text { Bayoumi et al. } \\
\text { [28] } \\
\text { (Canada) }\end{array}$ & 2008 & Prevention & $\begin{array}{l}\text { Strategy 2: alternative } \\
\text { foam mattress and 4- } \\
\text { hourly } \\
\text { turning/repositioning } \\
\text { schedules }\end{array}$ & $\begin{array}{l}\text { Standard } \\
\text { care }\end{array}$ & CUA & $\begin{array}{l}\text { Markov model } \\
\text { ( } 52 \text { health } \\
\text { states) }\end{array}$ & $\begin{array}{l}\text { Ontario } \\
\text { Ministry of } \\
\text { Health }\end{array}$ & Life time & $\Delta$ cost $/ \triangle Q A L Y$ & Cost-effective & $\begin{array}{l}\text { Robust (OW, } \\
\text { SA, PSA) }\end{array}$ \\
\hline $\begin{array}{l}\text { Makai et al. } \\
\text { [29] } \\
\text { (Netherlands) }\end{array}$ & 2010 & Prevention & $\begin{array}{l}\text { A quality } \\
\text { improvement } \\
\text { collaborative that } \\
\text { used evidence-based } \\
\text { preventive measures }\end{array}$ & $\begin{array}{l}\text { Before } \\
\text { implementa } \\
\text { tion of } \\
\text { protocol }\end{array}$ & CUA & $\begin{array}{l}\text { Markov model } \\
\text { (10 health } \\
\text { states) }\end{array}$ & Healthcare & 2 years & $\Delta$ cost $/ \triangle Q A L Y$ & Variable & Variable (PSA) \\
\hline $\begin{array}{l}\text { Mathiesen et } \\
\text { al. [30] } \\
\text { (Denmark) }\end{array}$ & 2013 & Prevention & $\begin{array}{l}\text { Guideline-based } \\
\text { Pressure Ulcer Bundle }\end{array}$ & $\begin{array}{l}\text { No specific } \\
\text { pressure } \\
\text { ulcer } \\
\text { prevention } \\
\text { strategy }\end{array}$ & CEA & Decision tree & $\begin{array}{l}\text { Public } \\
\text { healthcare }\end{array}$ & $\begin{array}{l}\text { Maximum } \\
\text { length of } \\
\text { healing time } \\
\text { for a pressure } \\
\text { ulcer plus } \\
\text { potential } \\
\text { complication }\end{array}$ & $\begin{array}{l}\Delta \text { cost and } \\
\text { number of } \\
\text { prevented } \\
\text { pressure ulcers } \\
\text { and the } \\
\text { number of } \\
\text { saved lives }\end{array}$ & Cost-saving & $\begin{array}{l}\text { Robust (OW, } \\
\text { PSA) }\end{array}$ \\
\hline $\begin{array}{l}\text { Padula et al. } \\
\text { [31] } \\
\text { (USA) }\end{array}$ & 2011 & Prevention & $\begin{array}{l}\text { Prevention based on } \\
\text { Wound, Ostomy, and } \\
\text { Continence Nurses } \\
\text { Society (WOCN) } \\
\text { guidelines }\end{array}$ & $\begin{array}{l}\text { Standard } \\
\text { care }\end{array}$ & CUA & $\begin{array}{l}\text { Semi-Markov } \\
\text { model ( } 6 \text { main } \\
\text { health states) }\end{array}$ & Societal & $\begin{array}{l}1 \text { year (QALYs } \\
\text { from remaining } \\
\text { life expectancy } \\
\text { were included) }\end{array}$ & $\Delta$ cost $/ \triangle Q A L Y$ & Cost-saving & $\begin{array}{l}\text { Robust (OW, } \\
\text { PSA) }\end{array}$ \\
\hline $\begin{array}{l}\text { Thomson et al. } \\
\text { [18] } \\
\text { (UK) }\end{array}$ & 1999 & Prevention & $\begin{array}{l}\text { A potential } \\
\text { prevention program }\end{array}$ & Current care & CBA & $\begin{array}{l}\text { Prevalence and } \\
\text { incidence } \\
\text { studies }\end{array}$ & $\begin{array}{l}\text { Not reported } \\
\text { (health system) }\end{array}$ & 1 year* & Net benefit & Cost-saving & Robust (OW) \\
\hline $\begin{array}{l}\text { Whitty et al. } \\
\text { [32] } \\
\text { (Australia) }\end{array}$ & 2017 & Prevention & $\begin{array}{l}\text { A care bundle based } \\
\text { on the Institute of } \\
\text { Healthcare } \\
\text { Improvement } \\
\text { recommendations } \\
\text { and is evidence-based }\end{array}$ & $\begin{array}{l}\text { Standard } \\
\text { care }\end{array}$ & CEA \& CBA & $\begin{array}{l}\text { Pragmatic } \\
\text { cluster- } \\
\text { randomised } \\
\text { trial }\end{array}$ & Health system & 4 weeks* & $\begin{array}{l}\text { cost per } \\
\text { pressure ulcer } \\
\text { avoided and } \\
\text { net monetary } \\
\text { benefit }\end{array}$ & $\begin{array}{l}\text { CEA: depend } \\
\text { on threshold; } \\
\text { CBA: not cost- } \\
\text { effective }\end{array}$ & Robust (OW) \\
\hline $\begin{array}{l}\text { Xakellis et al. } \\
\text { [33] } \\
\text { (UK and USA) }\end{array}$ & 1996 & Prevention & $\begin{array}{l}\text { Aggressive } \\
\text { interventive } \\
\text { interventions based } \\
\text { on 'Pressure ulcers in } \\
\text { adults: prediction and }\end{array}$ & $\begin{array}{l}\text { No } \\
\text { intervention }\end{array}$ & CEA & $\begin{array}{l}\text { Prospective } \\
\text { cohort study } \\
\text { and } \\
\text { retrospective } \\
\text { data audit } \\
\end{array}$ & $\begin{array}{l}\text { Not reported } \\
\text { (health system) }\end{array}$ & 3 months* & $\begin{array}{l}\text { Cost per ulcer- } \\
\text { free day }\end{array}$ & $\begin{array}{l}\text { Cost-saving for } \\
\text { higher risk } \\
\text { groups }\end{array}$ & NA \\
\hline
\end{tabular}




\begin{tabular}{|c|c|c|c|c|c|c|c|c|c|c|c|}
\hline & & & $\begin{array}{l}\text { prevention. Clinical } \\
\text { Practice Guideline, } \\
\text { Vol 3' }\end{array}$ & & & & & & & & \\
\hline $\begin{array}{l}\text { Xakellis et al. } \\
\text { [34] } \\
\text { (USA) }\end{array}$ & 1998 & Prevention & $\begin{array}{l}\text { Aggressive } \\
\text { interventive } \\
\text { interventions based } \\
\text { on 'Pressure ulcers in } \\
\text { adults: prediction and } \\
\text { prevention. Clinical } \\
\text { Practice Guideline, No } \\
\text { 3' and 'Treatment of } \\
\text { pressure ulcers. } \\
\text { Clinical Practice } \\
\text { Guideline, No 15' }\end{array}$ & $\begin{array}{l}\text { Before } \\
\text { implementa } \\
\text { tion }\end{array}$ & CEA & $\begin{array}{l}\text { Quasi- } \\
\text { experimental } \\
\text { study }\end{array}$ & $\begin{array}{l}\text { Health care } \\
\text { provider }\end{array}$ & 6 months* & $\begin{array}{l}\text { Cost per ulcer- } \\
\text { free day }\end{array}$ & Cost-saving & Variable (TW) \\
\hline
\end{tabular}


Table 3.Summary characteristics of economic evaluation studies for VLU

\begin{tabular}{|c|c|c|c|c|c|c|c|c|c|c|c|}
\hline $\begin{array}{l}\text { Author } \\
\text { (Setting) }\end{array}$ & $\begin{array}{l}\text { Publication } \\
\text { Year }\end{array}$ & Type of care & Intervention & Comparator & Analysis & Study design & Perspective & $\begin{array}{l}\text { Time } \\
\text { horizon/ } \\
\text { Study } \\
\text { period }\end{array}$ & $\begin{array}{l}\text { Outcome } \\
\text { measures }\end{array}$ & $\begin{array}{l}\text { Baseline } \\
\text { analysis }\end{array}$ & $\begin{array}{l}\text { Sensitivity } \\
\text { analysis }\end{array}$ \\
\hline $\begin{array}{l}\text { Bosanquet et } \\
\text { al. [35] } \\
\text { (UK) }\end{array}$ & 1993 & Treatment & $\begin{array}{l}\text { New service } \\
\text { provided by } \\
\text { community leg } \\
\text { clinics }\end{array}$ & $\begin{array}{l}\text { Standard care } \\
\text { before the new } \\
\text { services were set } \\
\text { up }\end{array}$ & CEA & $\begin{array}{l}\text { Quasi- } \\
\text { experimental } \\
\text { study }\end{array}$ & $\begin{array}{l}\text { Not reported } \\
\text { (health system) }\end{array}$ & $\begin{array}{l}\text { Not } \\
\text { reported }\end{array}$ & $\begin{array}{l}\text { Annual costs and } \\
\text { healing rate by } 12 \\
\text { weeks }\end{array}$ & Cost-saving & NA \\
\hline $\begin{array}{l}\text { Korn et al. [36] } \\
\text { (USA) }\end{array}$ & 2002 & Prevention & $\begin{array}{l}\text { Compression } \\
\text { stockings }+ \\
\text { education }\end{array}$ & No prophylaxis & CUA & $\begin{array}{l}\text { Markov model (4 } \\
\text { health states) }\end{array}$ & $\begin{array}{l}\text { Not reported } \\
\text { (health system } \\
\text { and societal) }\end{array}$ & Life time & $\Delta$ cost $/ \triangle Q A L Y$ & Cost-saving & Robust (OW) \\
\hline $\begin{array}{l}\text { McGuckin et } \\
\text { al. [37] } \\
\text { (UK and USA) }\end{array}$ & 2002 & $\begin{array}{l}\text { Diagnosis and } \\
\text { treatment }\end{array}$ & $\begin{array}{l}\text { Care based on } \\
\text { United States } \\
\text { Guidelines for the } \\
\text { Diagnosis and } \\
\text { Treatment of } \\
\text { Venous Leg } \\
\text { Ulcers and the } \\
\text { Oxfordshire } \\
\text { Guideline for } \\
\text { Venous Leg } \\
\text { Ulcers }\end{array}$ & $\begin{array}{l}\text { Retrospective with } \\
\text { no guidelines }\end{array}$ & CEA & $\begin{array}{l}\text { Prospective cohort } \\
\text { study and } \\
\text { retrospective data } \\
\text { audit }\end{array}$ & $\begin{array}{l}\text { Not reported } \\
\text { (health system) }\end{array}$ & $\begin{array}{l}\text { Not } \\
\text { reported }\end{array}$ & $\begin{array}{l}\text { Median costs and } \\
\text { healing rate }\end{array}$ & Cost-saving & NA \\
\hline $\begin{array}{l}\text { Simon et al. } \\
\text { [38] (UK) }\end{array}$ & 1996 & $\begin{array}{l}\text { Prevention } \\
\text { and treatment }\end{array}$ & $\begin{array}{l}\text { Community leg } \\
\text { ulcer clinic } \\
\text { includes } \\
\text { assessment, } \\
\text { compression } \\
\text { therapy for } \\
\text { treatment and } \\
\text { prevention }\end{array}$ & $\begin{array}{l}\text { No coordinated } \\
\text { attempt }\end{array}$ & CEA & $\begin{array}{l}\text { Retrospective data } \\
\text { audit }\end{array}$ & $\begin{array}{l}\text { Not reported } \\
\text { (health system) }\end{array}$ & 13 weeks* & $\begin{array}{l}\text { The proportion of } \\
\text { ulcerated limbs } \\
\text { completely } \\
\text { healed within } \\
\text { three months and } \\
\text { total cost of leg } \\
\text { care }\end{array}$ & Cost-saving & NA \\
\hline
\end{tabular}


Table 4. Summary characteristics of economic evaluation studies for mixed types of chronic wounds

\begin{tabular}{|c|c|c|c|c|c|c|c|c|c|c|c|c|}
\hline $\begin{array}{l}\text { Author } \\
\text { (Setting) }\end{array}$ & $\begin{array}{l}\text { Publication } \\
\text { Year }\end{array}$ & $\begin{array}{l}\text { Wound } \\
\text { type }\end{array}$ & $\begin{array}{l}\text { Type of } \\
\text { care }\end{array}$ & Intervention & Comparator & Analysis & Study design & Perspective & $\begin{array}{l}\text { Time horizon/ } \\
\text { Study period }\end{array}$ & $\begin{array}{l}\text { Outcome } \\
\text { measures }\end{array}$ & $\begin{array}{l}\text { Baseline } \\
\text { analysis }\end{array}$ & $\begin{array}{l}\text { Sensitivity } \\
\text { analysis }\end{array}$ \\
\hline $\begin{array}{l}\text { DaVanzo et } \\
\text { al. [39] } \\
\text { (USA) }\end{array}$ & 2010 & $\begin{array}{l}\text { DFU, PU, } \\
\text { VLU, AU }\end{array}$ & Treatment & $\begin{array}{l}\text { A specific } \\
\text { structured, } \\
\text { comprehensive } \\
\text { wound } \\
\text { management } \\
\text { protocol provided } \\
\text { by wound } \\
\text { management team }\end{array}$ & $\begin{array}{l}\text { Did not receive } \\
\text { protocol; did not } \\
\text { receive care from } \\
\text { wound management } \\
\text { team }\end{array}$ & CEA & $\begin{array}{l}\text { Retrospective } \\
\text { data audit }\end{array}$ & Medicare & 10 months* & $\begin{array}{l}\text { Health care } \\
\text { costs and } \\
\text { clinical } \\
\text { outcomes }\end{array}$ & Cost-saving & NA \\
\hline $\begin{array}{l}\text { Graves et al. } \\
\text { [40] } \\
\text { (Australia) }\end{array}$ & 2014 & $\begin{array}{l}\text { DFU, VLU, } \\
A U, \text { other } \\
\text { ulcers }\end{array}$ & $\begin{array}{l}\text { Treatment } \\
\text { and } \\
\text { prevention }\end{array}$ & $\begin{array}{l}\text { Specialist clinic that } \\
\text { follows evidence- } \\
\text { based care }\end{array}$ & $\begin{array}{l}\text { Retrospective phase: } \\
\text { prior to the admission } \\
\text { to the specialist clinic }\end{array}$ & CUA & $\begin{array}{l}\text { Markov } \\
\text { model ( } 3 \\
\text { health states) }\end{array}$ & $\begin{array}{l}\text { Not reported } \\
\text { (health } \\
\text { system) }\end{array}$ & 16 years & $\Delta$ cost $/ \triangle Q A L Y$ & $\begin{array}{l}\text { Reduced } \\
\text { cost }\end{array}$ & $\begin{array}{l}\text { Robust } \\
\text { (PSA) }\end{array}$ \\
\hline $\begin{array}{l}\text { Harris et al. } \\
{[41]} \\
\text { (Canda) }\end{array}$ & 2008 & $\begin{array}{l}\text { DFU, VLU, } \\
\text { PU, other } \\
\text { diabetic } \\
\text { ulcers }\end{array}$ & Treatment & $\begin{array}{l}\text { Specialty Service } \\
\text { Alone that practice } \\
\text { evidence-based } \\
\text { care }\end{array}$ & $\begin{array}{l}\text { Minimal involvement of } \\
\text { Specialty service with } \\
\text { Registered Nurse and } \\
\text { Registered Practical } \\
\text { Nurse that practice } \\
\text { current care }\end{array}$ & CEA & $\begin{array}{l}\text { Retrospective } \\
\text { data audit }\end{array}$ & $\begin{array}{l}\text { Not reported } \\
\text { (health } \\
\text { system) }\end{array}$ & $\begin{array}{l}2 \text { years } 3 \\
\text { months* }\end{array}$ & $\begin{array}{l}\text { Nursing visit } \\
\text { costs and time } \\
\text { to complete } \\
\text { closure of } \\
\text { chronic wounds }\end{array}$ & Cost-saving & NA \\
\hline $\begin{array}{l}\text { Rybak et al. } \\
\text { [42] } \\
\text { (Poland) }\end{array}$ & 2012 & Leg ulcers & Treatment & $\begin{array}{l}\text { New treatment } \\
\text { model based on } \\
\text { EWMA guidelines }\end{array}$ & Before implementation & CEA & $\begin{array}{l}\text { Quasi- } \\
\text { experimental } \\
\text { study }\end{array}$ & $\begin{array}{l}\text { Not reported } \\
\text { (health } \\
\text { system) }\end{array}$ & 30 weeks* & $\begin{array}{l}\text { Clinical } \\
\text { outcomes and } \\
\text { costs }\end{array}$ & Cost-saving & NA \\
\hline $\begin{array}{l}\text { Vu et al. [43] } \\
\text { (Australia) }\end{array}$ & 2007 & $\begin{array}{l}\text { leg ulcers } \\
\text { and PU }\end{array}$ & Treatment & $\begin{array}{l}\text { Multidisciplinary } \\
\text { team + standard } \\
\text { treatment protocol }\end{array}$ & $\begin{array}{l}\text { Usual care based on } \\
\text { 'The Residential Care } \\
\text { Manual' }\end{array}$ & CEA & $\begin{array}{l}\text { Pseudo- } \\
\text { randomized } \\
\text { cluster trial }\end{array}$ & Health system & 20 weeks* & $\begin{array}{l}\text { Cost-saving per } \\
\text { wound }\end{array}$ & Cost-saving & $\begin{array}{l}\text { Robust } \\
\text { (OW) }\end{array}$ \\
\hline
\end{tabular}




\subsubsection{Diabetic Foot Ulcer (DFU)}

Ragnarson Tennvall et al. [24], Rauner et al. [25] and Wu et al. [27] developed Markov models to evaluate the cost-effectiveness of optimal prevention of DFU based on the International consensus on diabetic foot [44]. Ragnarson Tennvall et al. [24] and Rauner et al. [25] found that optimal prevention would not be cost-effective for low-risk groups, which were supported by one-way sensitivity analysis. ICER was reported as the primary outcome in those three studies, which enabled direct comparison with other studies. Cardenas et al. [21] also investigated the cost-effectiveness of optimal prevention but with a decision tree model. Although the study reported that optimal prevention was cost-saving, the decision tree only estimated results within 1 year.

Optimal prevention and treatment of DFUs were assessed by Ortegon et al. [23], Rerkasem et al. [26] and Cheng et al. [22]. Rerkasem et al. [26] analysed retrospective audit data and reported that the Multidisciplinary Diabetic Foot Protocol was less expensive and gave patients a better quality of life. However, no sensitivity analysis was conducted to test the robustness of the outcome. Ortegon et al. [23] built a Markov model with comprehensive health states and estimated the costeffectiveness over a lifetime. The authors only conducted one-way sensitivity analysis and did not identify any limitations but argued that the probability for bias was small. The study by Cheng et al. [22] is the only study that performed probabilistic sensitivity analysis (PSA) and provided information on how confident decision makers could be about the findings. They showed that optimal care always had a higher probability (around 85-95\%) of being cost-effective compared with usual care regardless of the value of the willingness-to-pay threshold. But Cheng et al. [22] focused only on patients at high risk of DFUs. Moreover, although their study was conducted in an Australian setting, due to lack of local data, some model inputs were sourced from international studies.

\subsubsection{Pressure Ulcer (PU)}

All 8 included studies determined the cost-effectiveness of guideline-based prevention programs for PUs. Three studies conducted before 2000 did not apply decision-analytic models [18, 33, 34]. Even though the three studies demonstrated that prevention programs were cost-saving, the quality was low due to short study period and lack of comprehensive sensitivity analysis. Whitty et al. [32] evaluated a care bundle using data collected from a cluster randomised trial. They performed nonparametric bootstrapping to derive the cost-effectiveness acceptability curve, which suggested that the point estimate was highly uncertain. On the other hand, Padula et al. [31] and Mathiesen et al. [30] applied decision analytic models and performed PAS to assess the probability of prevention programs being cost-effective. The time horizon used in these two studies, however, was short, less than 1 year. As a result, the studies might not be useful for long-term decision-making.

Studies by Bayoumi et al. [28] and Makai et al. [29] provided strong evidence on the costeffectiveness of a PU prevention program. Bayoumi et al. obtained the effectiveness of the intervention from a systematic review and modelled costs and health outcomes associated with the intervention and control group over patients' lifetimes. In addition, comprehensive sensitivity analysis was performed. With PSA, authors concluded that the certainty that alternative foam mattress with turning/repositioning strategies were economically attractive was moderate. Makai et al. [29] built a Markov model to extrapolate results from a one-year observational study to an additional year. They tested three scenarios in which effectiveness of evidence-based prevention measures were (1) not sustained, (2) partially sustained, and (3) completely sustained. Results from PSA indicated that there was great uncertainty when the effectiveness of prevention methods was not sustained or partially sustained in the future, which reflected scenarios that could happen in the real world. 


\subsubsection{Venous Leg Ulcer (VLU)}

As a model-based economic evaluation, Korn et al. [36] conducted a thorough literature review to derive the effectiveness inputs for the model. The authors also applied discounting to future costs and health benefits. The model was run over the lifetime of affected individuals, which informed a long-term outcome. The baseline results were found to be robust given the one-way sensitivity analysis. However, without a PSA, it is difficult to determine how confident decision makers can be about the findings.

Bosanquet et al. [35], McGuckin et al. [37] and Simon et al. [38] did not develop economic models to extrapolate short-term findings beyond the study period. Neither did they conduct sensitivity analysis to test the uncertainty around parameters. When reporting the outcomes, they did not present the differences between groups. Moreover, limitations were not discussed in the study.

\subsection{Quality assessment}

Quality assessment of each study and reasons for a score of 0.5 are presented in Table 5 . None of the 24 included studies addressed every item listed in the CHEERS reporting checklist. The compliance with the CHEERS checklist ranged between $43 \%$ and $83 \%$. Studies published after $2013-$ when CHEERS statement was available - had compliance of at least $63 \%$.

Time horizon refers to the period over which costs and consequences are being evaluated. For economic evaluations that were not model-based, the study period was extracted as the time horizon. Although most studies evaluated costs and health outcomes within a timeframe, only one study stated why their choice of time horizon was appropriate for the study [25].

Economic evaluations that were not model-based did not apply discounting to costs and health consequences. For model-based studies, 3 provided background for the choice of discount rate [21, 22,29 ]. Mathiesen et al. [30] did not use discount rate because of the short time horizon. The rest of the model-based studies either did not justify the choice of discount rate or did not report the use of discount rate.

One thing worth noting is how measurement of effectiveness was obtained in each study. For economic evaluations that were not model-based, a score of 0.5 was given as they did not describe why the single study was a sufficient source of clinical effectiveness data. For model-based studies, three met the criteria with one conducting a systematic review [28] and two performing a thorough literature search $[27,36]$ to derive the effectiveness data for models, while the other 9 studies did not fully describe the methods used for identification of included studies and synthesis of clinical effectiveness data.

Authors of model-based studies all prepared tables for parameter values used in the model. However, two studies did not report the range of values [23, 22]; two did not report the values for transition probabilities $[25,29]$ and one did not present the values nor the range of model inputs [40]. While most parameter estimates were sourced from published literature, two evaluations directly used data collected from an existing trial or study $[29,40]$. With regards to the measurement and valuation of QoL outcomes, two studies derived utility estimates from population surveys $[28,40]$ and the remaining model-based evaluations used utility values from published literature.

Sixteen studies conducted sensitivity analyses to test the uncertainty of input parameters. The most commonly used method was one-way sensitivity analysis where one input was varied between a minimum and a maximum value at a time. Six studies conducted PSA and presented how confident decision makers could be about the baseline results [22, 27, 28, 30, 31, 40]. However, not all 
sensitivity analyses showed robust results. Xakellis et al. [34] found that the prevention strategy would be cost-saving except in the scenarios where treatment costs were reduced by an additional $50 \%$. Makai et al. [29] reported that if the effects from the prevention program could not be sustained, then the probability that the program was cost-effective would drop to $37 \%$ from $50 \%$ (totally sustained scenario). Only 4 studies characterized heterogeneity with 1 summarizing outcomes by age and risk groups [24], one evaluating cost-effectiveness for three age groups [22], one presenting ICERs for four risk groups [25] and 1 performing subgroup analysis by wound scores [33].

\subsection{Usefulness of economic evaluations}

We judged the usefulness of included studies to decision making based on data extraction and reporting quality assessment, and categorized them into 'strong', 'moderate' or 'limited' level as shown in Table 6. Having a high compliance score to CHEERS reporting checklist does not necessarily guarantee that the study is of great use to decision making. 
Table 5. Quality assessment using CHEERS statement

\begin{tabular}{|c|c|c|c|c|c|c|c|c|c|c|c|c|c|}
\hline & Study (Publication year) & $\begin{array}{l}\text { Cardenas } \\
\text { (2015) } \\
{[21]}\end{array}$ & $\begin{array}{l}\text { Cheng } \\
(2017) \\
{[22]}\end{array}$ & $\begin{array}{l}\text { Ortegon } \\
(2004) \\
{[23]}\end{array}$ & $\begin{array}{l}\text { Ragnarson } \\
\text { Tennvall } \\
\text { (2001)[24] }\end{array}$ & $\begin{array}{l}\text { Rauner } \\
(2005) \\
{[25]}\end{array}$ & $\begin{array}{l}\text { Rerkasem } \\
\text { (2009) } \\
{[26]}\end{array}$ & $\begin{array}{l}\text { Wu } \\
(2018) \\
{[27]}\end{array}$ & $\begin{array}{l}\text { Bayoumi } \\
(2008) \\
{[28]}\end{array}$ & $\begin{array}{l}\text { Makai } \\
(2010) \\
{[29]}\end{array}$ & $\begin{array}{l}\text { Mathiesen } \\
\text { (2013) } \\
{[30]}\end{array}$ & $\begin{array}{l}\text { Padula } \\
\text { (2011) } \\
{[31]}\end{array}$ & $\begin{array}{l}\text { Thomson } \\
\text { (1999) } \\
{[18]}\end{array}$ \\
\hline 1 & Title & 1 & 1 & 1 & 1 & $0.5^{\mathrm{a}}$ & $0.5^{\mathrm{a}}$ & 1 & 1 & 1 & 1 & 1 & $0.5^{\mathrm{a}}$ \\
\hline 3 & Background and objectives & 1 & 1 & 1 & 1 & 1 & 1 & 1 & 1 & 1 & 1 & 1 & 1 \\
\hline 4 & Target population and subgroups & 1 & 1 & 1 & 1 & 1 & 1 & 1 & 1 & 1 & 0 & 0 & 1 \\
\hline 5 & Setting and location & 1 & 1 & 1 & 1 & 1 & 1 & 1 & 1 & 1 & 1 & 1 & 1 \\
\hline 7 & Comparators & 1 & 1 & 1 & 1 & 1 & 1 & 1 & $0.5^{\mathrm{d}}$ & $0.5^{d}$ & 1 & 1 & $0.5^{\mathrm{d}}$ \\
\hline 8 & Time horizon & $0.5^{\mathrm{e}}$ & $0.5^{\mathrm{e}}$ & $0.5^{\mathrm{e}}$ & $0.5^{\mathrm{e}}$ & 1 & $0.5^{\mathrm{e}}$ & $0.5^{\mathrm{e}}$ & $0.5^{\mathrm{e}}$ & $0.5^{\mathrm{e}}$ & $0.5^{\mathrm{e}}$ & $0.5^{\mathrm{e}}$ & $0.5^{\mathrm{e}}$ \\
\hline 9 & Discount rate & 1 & 1 & $0.5^{\mathrm{e}}$ & $0.5^{\mathrm{e}}$ & $0.5^{\mathrm{e}}$ & 0 & 1 & $0.5^{\mathrm{e}}$ & 1 & $0.5^{f}$ & $0.5^{\mathrm{e}}$ & 0 \\
\hline 10 & Choice of health outcomes & $0.5^{\mathrm{g}}$ & $0.5^{\mathrm{g}}$ & $0.5^{\mathrm{g}}$ & $0.5^{\mathrm{g}}$ & 1 & $0.5^{\mathrm{g}}$ & 1 & 1 & 1 & 1 & $0.5^{\mathrm{g}}$ & 1 \\
\hline 11 & Measurement of effectiveness & $0.5^{i}$ & $0.5^{i}$ & $0.5^{i}$ & $0.5^{i}$ & $0.5^{i}$ & $0.5^{\mathrm{h}}$ & 1 & 1 & $0.5^{\mathrm{h}}$ & $0.5^{i}$ & $0.5^{\mathrm{i}}$ & $0.5^{\mathrm{h}}$ \\
\hline 12 & $\begin{array}{l}\text { Measurement and valuation of } \\
\text { preference-based outcomes }\end{array}$ & NA & NA & NA & NA & NA & 1 & NA & 1 & NA & NA & NA & NA \\
\hline 14 & Currency, price date, and conversion & 1 & 1 & 1 & 1 & 1 & 1 & $0.5^{\mathrm{s}}$ & $0.5^{\mathrm{s}}$ & $0.5^{\mathrm{s}}$ & 1 & 1 & 0 \\
\hline 15 & Choice of model & $0.5^{\mathrm{j}}$ & 1 & 1 & 1 & 1 & NA & $0.5^{j}$ & $0.5^{j}$ & $0.5^{\mathrm{k}}$ & $0.5^{\mathrm{j}}$ & 1 & NA \\
\hline 16 & Assumptions & 1 & 1 & 0 & 1 & 1 & NA & 1 & 1 & 1 & 1 & 1 & NA \\
\hline 17 & Analytical methods & $0.5^{\mathrm{u}}$ & $0.5^{\mathrm{u}}$ & $0.5^{\mathrm{u}}$ & 1 & $0.5^{\mathrm{u}}$ & NA & $0.5^{\mathrm{u}}$ & 1 & $0.5^{\mathrm{u}}$ & $0.5^{\mathrm{u}}$ & 1 & NA \\
\hline 18 & Study parameters & 1 & $0.5^{1}$ & $0.5^{\prime}$ & 1 & $0.5^{1}$ & 0 & 1 & 1 & $0.5^{\prime}$ & 1 & 1 & $0.5^{\prime}$ \\
\hline 19 & Incremental costs and outcomes & $0.5^{n}$ & 1 & 1 & 1 & 0 & $0.5^{\mathrm{m}}$ & 1 & $0.5^{n}$ & $0.5^{n}$ & 1 & 1 & $0.5^{n}$ \\
\hline 20 & Characterising uncertainty & $0.5^{\circ}$ & 1 & $0.5^{\circ}$ & $0.5^{\circ}$ & $0.5^{\circ}$ & 0 & 1 & 1 & 1 & 1 & 1 & $0.5^{p}$ \\
\hline 21 & Characterising heterogeneity & 0 & 1 & 0 & 1 & 0 & 0 & 0 & 0 & 0 & 0 & 0 & 0 \\
\hline 22 & $\begin{array}{l}\text { Study findings, limitations, } \\
\text { generalisability, and current knowledge }\end{array}$ & 1 & 1 & $0.5^{q}$ & 1 & 1 & 1 & 1 & 1 & 1 & 1 & 1 & 1 \\
\hline 23 & Source of funding & 1 & 0 & 0 & $0.5^{r}$ & 0 & $0.5^{r}$ & 0 & $0.5^{r}$ & 0 & 1 & 0 & 0 \\
\hline \multirow[t]{2}{*}{24} & Conflicts of interest & 1 & 0 & 0 & 0 & 0 & 1 & 1 & 0 & 1 & 1 & 0 & 0 \\
\hline & Compliance with the CHEERS checklist* & $78 \%$ & $78 \%$ & $61 \%$ & $76 \%$ & $65 \%$ & $57 \%$ & $83 \%$ & $79 \%$ & $74 \%$ & $80 \%$ & $74 \%$ & $53 \%$ \\
\hline
\end{tabular}


Table 5. Quality assessment using CHEERS statement (continued)

\begin{tabular}{|c|c|c|c|c|c|c|c|c|c|c|c|c|c|}
\hline & Study (Publication year) & $\begin{array}{l}\text { Whitty } \\
\text { (2017) } \\
{[32]}\end{array}$ & $\begin{array}{l}\text { Xakellis } \\
\text { (1996) } \\
{[33]}\end{array}$ & $\begin{array}{l}\text { Xakellis } \\
(1998) \\
{[34]}\end{array}$ & $\begin{array}{l}\text { Bosanquet } \\
\text { (1993) } \\
{[35]}\end{array}$ & $\begin{array}{l}\text { Korn } \\
(2002) \\
{[36]}\end{array}$ & $\begin{array}{l}\text { McGuckin } \\
(2002) \\
{[37]}\end{array}$ & $\begin{array}{l}\text { Simon } \\
(1996) \\
{[38]}\end{array}$ & $\begin{array}{l}\text { DaVanzo } \\
(2010) \\
{[39]}\end{array}$ & $\begin{array}{l}\text { Graves } \\
(2014) \\
{[40]}\end{array}$ & $\begin{array}{l}\text { Harris } \\
(2008) \\
{[41]}\end{array}$ & $\begin{array}{l}\text { Rybak } \\
(2012) \\
{[42]}\end{array}$ & $\begin{array}{l}\mathrm{Vu} \\
(2007) \\
{[43]}\end{array}$ \\
\hline 1 & Title & 1 & 1 & 1 & 1 & 0 & 0 & $0.5^{\mathrm{a}}$ & $0.5^{\mathrm{a}}$ & $0.5^{a}$ & 1 & $0.5^{a}$ & 1 \\
\hline 2 & Abstract & 1 & $0.5^{\mathrm{b}}$ & $0.5^{\mathrm{b}}$ & $0.5^{\mathrm{b}}$ & 1 & $0.5^{b}$ & $0.5^{b}$ & $0.5^{\mathrm{b}}$ & $0.5^{b}$ & $0.5^{\mathrm{b}}$ & $0.5^{\mathrm{b}}$ & $0.5^{b}$ \\
\hline 3 & Background and objectives & 1 & 1 & 1 & 1 & 1 & 1 & 1 & 1 & 1 & 1 & 1 & 1 \\
\hline 4 & Target population and subgroups & 1 & 1 & 1 & 1 & 1 & 1 & 1 & 1 & 1 & 1 & 1 & 1 \\
\hline 5 & Setting and location & 1 & 1 & 1 & 1 & 1 & 1 & 1 & 1 & 1 & 1 & 1 & 1 \\
\hline 6 & Study perspective & 1 & 0 & 1 & 0 & 0 & 0 & 0 & 1 & 0 & 0 & 0 & 1 \\
\hline 7 & Comparators & 1 & 1 & 1 & $0.5^{d}$ & $0.5^{d}$ & 1 & $0.5^{d}$ & $0.5^{d}$ & $0.5^{d}$ & $0.5^{d}$ & 1 & $0.5^{d}$ \\
\hline 8 & Time horizon & $0.5^{\mathrm{e}}$ & $0.5^{\mathrm{e}}$ & $0.5^{\mathrm{e}}$ & 0 & $0.5^{\mathrm{e}}$ & 0 & $0.5^{\mathrm{e}}$ & $0.5^{\mathrm{e}}$ & $0.5^{\mathrm{e}}$ & $0.5^{\mathrm{e}}$ & $0.5^{\mathrm{e}}$ & $0.5^{\mathrm{e}}$ \\
\hline 9 & Discount rate & 0 & 0 & 0 & 0 & 1 & 0 & 0 & 0 & 0 & 0 & 0 & 0 \\
\hline 10 & Choice of health outcomes & 1 & 1 & 1 & $0.5^{\mathrm{g}}$ & $0.5^{\mathrm{g}}$ & 1 & $0.5^{\mathrm{g}}$ & 1 & 1 & 1 & 1 & $0.5^{\mathrm{g}}$ \\
\hline 11 & Measurement of effectiveness & $0.5^{\mathrm{h}}$ & $0.5^{\mathrm{h}}$ & $0.5^{\mathrm{h}}$ & $0.5^{\mathrm{h}}$ & 1 & $0.5^{\mathrm{h}}$ & $0.5^{\mathrm{h}}$ & $0.5^{\mathrm{h}}$ & $0.5^{\mathrm{h}}$ & $0.5^{\mathrm{h}}$ & $0.5^{\mathrm{h}}$ & $0.5^{\mathrm{h}}$ \\
\hline 12 & $\begin{array}{l}\text { Measurement and valuation of } \\
\text { preference based outcomes }\end{array}$ & NA & NA & NA & NA & NA & NA & NA & NA & 1 & NA & NA & NA \\
\hline 13 & Estimating resources and costs & 1 & 1 & 1 & 1 & 1 & 1 & 1 & 1 & 1 & 1 & 1 & 1 \\
\hline 14 & Currency, price date, and conversion & 1 & 0 & 0 & 1 & 1 & 0 & 1 & 1 & $0.5^{\mathrm{s}}$ & 1 & 0 & 1 \\
\hline 15 & Choice of model & NA & NA & NA & NA & $0.5^{j}$ & NA & NA & NA & $0.5^{j}$ & NA & NA & NA \\
\hline 16 & Assumptions & NA & NA & NA & NA & 1 & NA & NA & NA & 1 & NA & NA & NA \\
\hline 17 & Analytical methods & NA & NA & NA & NA & $0.5^{\mathrm{u}}$ & NA & NA & NA & $0.5^{\mathrm{u}}$ & NA & NA & NA \\
\hline 18 & Study parameters & $0.5^{1}$ & $0.5^{1}$ & 0 & $0.5^{\prime}$ & 1 & $0.5^{1}$ & $0.5^{1}$ & 1 & 0 & $0.5^{1}$ & 0 & $0.5^{\prime}$ \\
\hline 19 & Incremental costs and outcomes & 1 & $0.5^{\mathrm{m}}$ & 0 & $0.5^{\mathrm{m}}$ & 1 & $0.5^{\mathrm{m}}$ & $0.5^{\mathrm{m}}$ & 1 & 1 & 1 & $0.5^{\mathrm{m}}$ & $0.5^{\mathrm{m}}$ \\
\hline 20 & Characterising uncertainty & $0.5^{\mathrm{t}}$ & 0 & 1 & 0 & 1 & 0 & 0 & 0 & 1 & 0 & 0 & 0 \\
\hline 21 & Characterising heterogeneity & 0 & 1 & 0 & 0 & 0 & 0 & 0 & 0 & 0 & 0 & 0 & 0 \\
\hline 22 & $\begin{array}{l}\text { Study findings, limitations, } \\
\text { generalisability, and current knowledge }\end{array}$ & 1 & 1 & 1 & $0.5^{9}$ & $0.5^{q}$ & $0.5^{9}$ & $0.5^{q}$ & 1 & 1 & 1 & $0.5^{q}$ & 1 \\
\hline 23 & Source of funding & $0.5^{r}$ & $0.5^{r}$ & $0.5^{r}$ & 0 & 0 & 0 & $0.5^{r}$ & 1 & $0.5^{r}$ & $0.5^{r}$ & 0 & $0.5^{r}$ \\
\hline \multirow[t]{2}{*}{24} & Conflicts of interest & 0 & 0 & 0 & 0 & 0 & 0 & 1 & 1 & 0 & 0 & 0 & 1 \\
\hline & Compliance with the CHEERS checklist* & $73 \%$ & $60 \%$ & $60 \%$ & $48 \%$ & $65 \%$ & $43 \%$ & $55 \%$ & $73 \%$ & $63 \%$ & $63 \%$ & $45 \%$ & $65 \%$ \\
\hline
\end{tabular}


a. did not identify the study as an economic evaluation or use more specific terms

b. did not report uncertainty analysis

c. did not include inputs

d. did not explicitly mention the intervention was guideline-based

e. did not state why

f. did not use discount rate but gave reasons

g. did not state the relevance

h. did not state why the single study was a sufficient source of clinical effectiveness data

i. did not describe the methods used for identification of included studies and synthesis of clinical effectiveness data

j. did not give reasons for the specific type of decision-analytical model used

k. did not give reasons for the specific type of decision-analytical model used nor show the model structure

I. did not report values, ranges, references for all parameters

m. did not report differences

n. did not report mean values for the main categories of estimated costs and outcomes of interest

o. not all input parameters were tested for uncertainty

p. did not separate sensitivity analysis from baseline analysis

q. did not discuss limitations

r. did not describe the role of the funder in the identification, design, conduct, and reporting of the analysis

s. did not include reference year

t. applied non-parametric bootstrapping

u. only applied face validation of the model or no validation

$\mathrm{NA}=$ not applicable

*Fully meeting the criteria contributed 1 to the numerator and partially meeting the criteria contributed 0.5 to the numerator 


\begin{tabular}{|c|c|c|}
\hline Study & Level of usefulness & Primary reasons \\
\hline Bayoumi et al. [28] & Strong & $\begin{array}{l}\text { Life-time horizon; applied model calibration and full sensitivity } \\
\text { analysis }\end{array}$ \\
\hline Cardenas et al. [21] & Moderate & $\begin{array}{l}\text { Outcome measures used; lack of probabilistic sensitivity } \\
\text { analysis }\end{array}$ \\
\hline Cheng et al.[22] & Moderate & Lack of appropriate model validation; no indirect costs \\
\hline Ortegon et al. [23] & Moderate & Lack of probabilistic sensitivity analysis; no indirect costs \\
\hline Ragnarson Tennvall et al. [24] & Moderate & Lack of probabilistic sensitivity analysis; no indirect costs \\
\hline Rauner et al. [25] & Moderate & Lack of probabilistic sensitivity analysis; no indirect costs \\
\hline Wu et al. [27] & Moderate & Lack of appropriate model validation; no indirect costs \\
\hline Makai et al. [29] & Moderate & Lack of appropriate model validation; no indirect costs \\
\hline Mathiesen et al. [30] & Moderate & Time horizon and outcome measures used; no indirect costs \\
\hline Whitty et al. [32] & Moderate & Lack of probabilistic sensitivity analysis; short time horizon \\
\hline Korn et al. [36] & Moderate & Lack of probabilistic sensitivity analysis \\
\hline Graves et al. [40] & Moderate & Lack of appropriate model validation; no indirect costs \\
\hline Vu et al. [43] & Moderate & Lack of probabilistic sensitivity analysis; short time horizon \\
\hline Rerkasem et al. [26] & Limited & Study design; lack of sensitivity analysis \\
\hline Padula et al. [31] & Limited & $\begin{array}{l}\text { One-year time horizon but included QALYs over remaining life } \\
\text { expectancy as final reward }\end{array}$ \\
\hline Thomson et al. [18] & Limited & Study design; lack of probabilistic sensitivity analysis \\
\hline Xakellis et al. [33] & Limited & Outcome measures used; short time horizon; no indirect costs \\
\hline Xakellis et al. [34] & Limited & Outcome measures used; short time horizon; no indirect costs \\
\hline Bosanquet et al. [35] & Limited & $\begin{array}{l}\text { Different time frame for costs and health outcomes; lack of } \\
\text { sensitivity analysis }\end{array}$ \\
\hline McGuckin et al. [37] & Limited & $\begin{array}{l}\text { Outcome measures used; lack of sensitivity analysis; no indirect } \\
\text { costs }\end{array}$ \\
\hline Simon et al. [38] & Limited & Lack of sensitivity analysis; short time horizon; no indirect costs \\
\hline DaVanzo et al. [39] & Limited & Lack of sensitivity analysis; short time horizon; no indirect costs \\
\hline Harris et al. [41] & Limited & Outcome measures used; lack of sensitivity analysis \\
\hline Rybak et al. [42] & Limited & Lack of sensitivity analysis; short time horizon; no indirect costs \\
\hline
\end{tabular}




\section{DISCUSSION}

We reviewed existing full economic evaluation studies of guideline-based care and found that guideline-based care may be cost-saving or cost-effective in most circumstances. In other words, providing better wound management based on guidelines would improve patient outcomes at a lower cost or at some acceptable additional cost. In the absence of perfect information, those economic evaluation studies can give decision makers the confidence to promote guideline-based care. However, the level of confidence varies greatly by the study design and the quality of economic evaluations.

\subsection{General issues}

\subsubsection{Time horizon}

Researchers tended to report short-term results if they simply analysed data from retrospective data audit, before/after study, or randomised controlled trials. They often chose healing as the event of interest and endpoint. But chronic wounds are highly likely to recur. Studies with a short time horizon would fail to capture benefits or identify losses in the future. As a result, information generated from this type of economic evaluation is of limited use to decision makers to incorporate into long-term planning.

\subsubsection{Perspective and indirect cost}

Some studies did not state what economic perspective the studies were based on. The perspective of an economic evaluation plays an important role, because it determines whether the study should include the impact of a program on the whole society or just on those directly involved. Failure to report the perspective again limited the usefulness of these studies to decision makers. Most studies were conducted from a health system perspective and calculated the direct costs to the health system. However, omitting indirect cost such as patient out-of-pocket costs would fail to inform whether the decision to adopt a certain policy would benefit the whole society. Only four studies were conducted from a societal perspective $[21,36,31,26]$ and three of them included opportunity costs from lost productivity $[21,36,26]$. One study claimed to use a societal perspective but simply assumed additional $25 \%$ unforeseen costs [31].

\subsubsection{Sensitivity analysis}

The level of confidence in economic evaluation studies can be boosted by sensitivity analyses. In this review, we found that 14 out of 22 included studies performed sensitivity analysis to test the uncertainty around study variables. Studies that performed PSA provided the strongest evidence on the robustness of study findings, since PSA explicitly demonstrates the probability that a program is cost-effective. Decision makers would find this type of information useful when they want to avoid investing in programs that are unlikely to be good value for money.

\subsubsection{Wound types}

In this systematic review, we also found that the level of evidence varies by wound type. There is strong evidence showing that guideline-based care for DFU and PU is cost-effective or cost-saving, while evidence is weak in terms of guideline-based care for VLU. We identified 4 studies that evaluated health outcomes and costs of implementing good practice for VLU, but those studies are not up-to-date and the quality is not satisfactory. Moreover, although AU is one type of chronic wound, we couldn't identify any study that specifically conducted economic evaluation of guidelinebased care for AU. Thus, more economic evaluation studies are required to raise the evidence level. 


\subsection{Model-based studies}

Several studies in this review applied Markov models to estimate long-term patient outcomes and costs. It seems that model-based economic evaluation fully described the disease progression and provided better information. But not all model-based economic evaluations attained satisfactory quality scores.

\subsubsection{Model structure and assumption}

When modelling techniques are applied to healthcare decision making, it has been recommended that models represent disease processes appropriately and addresses the decision problem [45]. Of the 12 reviewed model-based studies, health states or clinical events closely related to ulcer progressing and costs were modelled and presented clearly in figures in most studies. But one study only summarized transitions between health states in tables [25] and one study did not provide any table or figure for model structure [29]. Assumptions around model structure have an impact on model prediction. For example, studies that used Markov model to evaluate cost-effectiveness of managing DFU all assumed that patients would not develop another ulcer or have amputation after one major amputation. The assumption may be reasonable for models with shorter time horizon. But for models with a lifetime time horizon [23, 27], the total costs of managing DFU could be underestimated as it is possible for patients to experience recurrence and another amputation.

\subsubsection{Model validation and calibration}

Comprehensive validation and calibration could help improve accuracy and credibility of decisionanalytic models [45]. But a lack of model validation and calibration was observed among modelbased economic evaluations reviewed in this study despite the recommendations on reporting model performance evaluation. Two studies did not provide information on validation of their Markov models probably because the models were built based on trial data [29, 40]. One study applied internal validation, comparing ulcer incidence and amputation incidence derived from the model with numbers used as model inputs [23]. Two studies conducted external validation by comparing model outputs with observations that were not used in model development[24, 31]. One study performed calibration to ensure that the input incidence produced observed prevalence [28]. The other 8 studies applied basic face validity such as comparing model outputs with other published studies to justify their results were sensible.

\subsubsection{Quality of model inputs}

Although economic evaluation is not expected to produce perfect prediction of future costs and health outcomes, the use of poor-quality data sources will lead to concerns over the credibility of model outputs. All model-based studies reviewed conducted the evaluation within the national setting. However, lack of local data sources was identified as a limitation in almost all studies that resorted to international literature to some extent. Using epidemiological, costs or QoL parameters generated from a different population as key model inputs may lead to underestimation or overestimation of health outcomes and costs. Another issue related to the quality of model inputs is data synthesis. For models built on a single trial, the issue is whether the single study acts as sufficient source of data. Markov models are data driven, usually requiring synthesis of data from different sources. We found that only 3 studies fully describe the methods used for identification of included studies and synthesis of clinical effectiveness data [27, 28, 36]. Key epidemiological and clinical parameters used in model-based studies often originated from sources of varying quality, ranging from experimental studies to expert opinion. 


\subsection{Limitations}

This review has four main limitations. First, we may have missed some economic evaluation studies despite the use of broad search terms. Second, our findings may be subject to publication bias as significant results are more likely to be published. It might be more difficult to publish studies that report that guideline-based wound care was not cost-effective or cost-saving or the authors might be less willing to submit these results for publication. Thus, readers should bear in mind that it is possible that guideline-based care may not always bring economic benefits. Third, we assessed the quality of studies and strength of evidence based on judgment. Finally, our assessment of study quality using the CHEERS checklist may reflect the way evaluations are reported rather than conducted. Therefore, the conclusions should be interpreted with caution.

\section{CONCLUSION}

The economic evaluation studies identified by this systematic review suggested that adopting guideline-based care may be a cost-effective or cost-saving strategy for patients affected by chronic wounds. There are variations in the quality of studies and the information presented is not always useful to assist decision making. Better information and higher-quality studies may increase decision makers' confidence to promote guideline-based wound care in the future. We also suggest that future economic evaluations in this field conform more closely to CHEERS reporting guidelines.

\section{Compliance with Ethical Standards}

Data Availability Statement: All data generated or analysed during this study are included in this published article (and its supplementary information files).

Competing interests: The authors, QC, NG and REP declare that they have no competing interests.

Funding: This research is part of Qinglu Cheng's PhD project which is funded by Queensland University of Technology Postgraduate Research Award and Wound Management Innovation Cooperative Research Centre Top Up Scholarship. The funders had no role in the design of the study and collection, analysis, and interpretation of data and in writing the manuscript or submitting for publication.

Acknowledgements: The authors would like to acknowledge the support of the Australian Government's Cooperative Research Centres Program. The Wound Management Innovation Cooperative Research Centre (WMI CRC) receives funding from the Australian Government, Curtin University of Technology, Queensland University of Technology, Smith \& Nephew Proprietary Limited, University of South Australia, Wounds Australia Ltd, Blue Care, the Department of Health South Australia, the Department of Health and Human Services Victoria, Ego Pharmaceuticals Pty Ltd, Metropolitan Health Service, Royal District Nursing Service Limited, Royal Melbourne Institute of Technology, Swinburne University of Technology, Silver Chain Group, Paul Hartmann Pty Ltd, MöInlycke Health Care Pty Ltd, KCI Medical Australia Pty Ltd, 3M Australia Pty Ltd, University of Queensland, University of Tasmania, University of Western Australia, University of Melbourne and Capital Health Network.

Author Contributions: All authors contributed to the development of this study. QC led the literature search. Full-text articles were assessed by QC and REP for eligibility. REP and NG supervised the 
study. All authors were actively involved in editing the drafts of the manuscript and interpreting the results. All authors read and approved the final manuscript. 


\section{Reference}

1. Nunan R, Harding KG, Martin P. Clinical challenges of chronic wounds: searching for an optimal animal model to recapitulate their complexity. Disease Models \& Mechanisms. 2014;7(11):1205-13. doi:10.1242/dmm.016782.

2. Sussman G. Ulcer dressings and management. Australian Family Physician. 2014;43:588-92.

3. Sen CK, Gordillo GM, Roy S, Kirsner R, Lambert L, Hunt TK et al. Human Skin Wounds: A Major and Snowballing Threat to Public Health and the Economy. Wound repair and regeneration : official publication of the Wound Healing Society [and] the European Tissue Repair Society. 2009;17(6):76371. doi:10.1111/j.1524-475X.2009.00543.x.

4. Posnett J, Franks PJ. The burden of chronic wounds in the UK. Nursing times. 2008;104(3):44-5.

5. Green J, Jester R, McKinley R, Pooler A. The impact of chronic venous leg ulcers: a systematic review. Journal of wound care. 2014;23(12):601-12. doi:10.12968/jowc.2014.23.12.601.

6. Goodridge D, Trepman E, Embil JM. Health-related quality of life in diabetic patients with foot ulcers: literature review. Journal of wound, ostomy, and continence nursing : official publication of The Wound, Ostomy and Continence Nurses Society. 2005;32(6):368-77.

7. Gorecki C, Brown JM, Nelson EA, Briggs M, Schoonhoven L, Dealey C et al. Impact of pressure ulcers on quality of life in older patients: a systematic review. Journal of the American Geriatrics Society. 2009;57(7):1175-83. doi:10.1111/j.1532-5415.2009.02307.x.

8. Graves N, Zheng H. Modelling the direct health care costs of chronic wounds in Australia. Wound Practice \& Research: Journal of the Australian Wound Management Association. 2014;22(1, Mar 2014):20-4, 6-33.

9. Nussbaum SR, Carter MJ, Fife CE, DaVanzo J, Haught R, Nusgart M et al. An Economic Evaluation of the Impact, Cost, and Medicare Policy Implications of Chronic Nonhealing Wounds. Value Health. 2018;21(1):27-32. doi:10.1016/j.jval.2017.07.007.

10. Bakker K, Apelqvist J, Lipsky BA, Van Netten JJ. The 2015 IWGDF guidance documents on prevention and management of foot problems in diabetes: development of an evidence-based global consensus. Diabetes Metab Res Rev. 2016;32 Suppl 1:2-6. doi:10.1002/dmrr.2694. 11. Australian Wound Management Association, New Zealand Wound Care Society. Australian and New Zealand Clinical Practice Guideline for Prevention and Management of Venous Leg Ulcers. 2011. 12. National Pressure Ulcer Advisory Panel, European Pressure Ulcer Advisory Panel, Pan Pacific Pressure Injury Alliance. Prevention and Treatment of Pressure Ulcers: Clinical Practice Guideline 2014.

13. Norman RE, Gibb M, Dyer A, Prentice J, Yelland S, Cheng $Q$ et al. Improved wound management at lower cost: a sensible goal for Australia. International wound journal. 2016;13(3):303-16. doi:10.1111/iwj.12538.

14. Edwards H, Finlayson K, Courtney M, Graves N, Gibb M, Parker C. Health service pathways for patients with chronic leg ulcers: identifying effective pathways for facilitation of evidence based wound care. BMC Health Services Research. 2013;13(1):86. doi:10.1186/1472-6963-13-86.

15. Harrison MB, Graham ID, Lorimer K, Friedberg E, Pierscianowski T, Brandys T. Leg-ulcer care in the community, before and after implementation of an evidence-based service. CMAJ : Canadian Medical Association Journal. 2005;172(11):1447-52. doi:10.1503/cmaj.1041441.

16. Carter MJ. Economic evaluations of guideline-based or strategic interventions for the prevention or treatment of chronic wounds. Applied health economics and health policy. 2014;12(4):373-89. doi:10.1007/s40258-014-0094-9.

17. Gordon L, Edwards H, Courtney M, Finlayson K, Shuter P, Lindsay E. A cost-effectiveness analysis of two community models of care for patients with venous leg ulcers. Journal of wound care. 2006;15(8):348-53. doi:10.12968/jowc.2006.15.8.26942.

18. Thomson JS, Brooks RG. The economics of preventing and treating pressure ulcers: a pilot study. Journal of wound care. 1999;8(6):312-6. 
19. Moher D, Liberati A, Tetzlaff J, Altman DG. Preferred reporting items for systematic reviews and meta-analyses: the PRISMA statement. BMJ. 2009;339. doi:10.1136/bmj.b2535.

20. Husereau D, Drummond M, Petrou S, Carswell C, Moher D, Greenberg D et al. Consolidated Health Economic Evaluation Reporting Standards (CHEERS) statement. BMJ : British Medical Journal. 2013;346. doi:10.1136/bmj.f1049.

21. Cardenas MK, Mirelman AJ, Galvin CJ, Lazo-Porras M, Pinto M, Miranda JJ et al. The cost of illness attributable to diabetic foot and cost-effectiveness of secondary prevention in Peru. BMC Health Serv Res. 2015;15:483. doi:10.1186/s12913-015-1141-4.

22. Cheng Q, Lazzarini PA, Gibb M, Derhy PH, Kinnear EM, Burn E et al. A cost-effectiveness analysis of optimal care for diabetic foot ulcers in Australia. International wound journal. 2017;14(4):616-28. doi:10.1111/iwj.12653.

23. Ortegon MM, Redekop WK, Niessen LW. Cost-Effectiveness of Prevention and Treatment of the Diabetic Foot: A Markov analysis. Diabetes Care. 2004;27(4):901-7. doi:10.2337/diacare.27.4.901.

24. Ragnarson Tennvall G, Apelqvist J. Prevention of diabetes-related foot ulcers and amputations: a cost-utility analysis based on Markov model simulations. Diabetologia. 2001;44(11):2077-87. doi:10.1007/s001250100013.

25. Rauner MS, Heidenberger K, Pesendorfer EM. Model-based evaluation of diabetic foot prevention strategies in Austria. Health Care Management Science. 2005;8(4):253-65.

26. Rerkasem K, Kosachunhanun N, Tongprasert S, Guntawongwan K. A multidisciplinary diabetic foot protocol at chiang mai university hospital: Cost and quality of life. International Journal of Lower Extremity Wounds. 2009;8(3):153-6. doi:10.1177/1534734609344143.

27. Wu B, Wan X, Ma J. Cost-effectiveness of prevention and management of diabetic foot ulcer and amputation in a health resource-limited setting. J Diabetes. 2018;10(4):320-7. doi:10.1111/17530407.12612.

28. Bayoumi A, John-Baptiste A, Chen MH, Chen W, Farahati F, Krahn M et al. The cost-effectiveness of prevention strategies for pressure ulcers in long-term care homes in Ontario: projections of the Ontario pressure ulcer model. Toronto: Toronto Health Economics and Technology Assessment Collaborative; 2008.

29. Makai P, Koopmanschap M, Bal R, Nieboer AP. Cost-effectiveness of a pressure ulcer quality collaborative. Cost Effectiveness and Resource Allocation. 2010;8. doi:10.1186/1478-7547-8-11. 30. Mathiesen ASM, Norgaard K, Andersen MFB, Moller KM, Ehlers LH. Are labour-intensive efforts to prevent pressure ulcers cost-effective? Journal of Medical Economics. 2013;16(10):1238-45. doi:10.3111/13696998.2013.832256.

31. Padula WV, Mishra MK, Makic MBF, Sullivan PW. Improving the quality of pressure ulcer care with prevention a cost-effectiveness analysis. Medical Care. 2011;49(4):385-92.

32. Whitty JA, McInnes E, Bucknall T, Webster J, Gillespie BM, Banks M et al. The cost-effectiveness of a patient centred pressure ulcer prevention care bundle: Findings from the INTACT cluster randomised trial. Int J Nurs Stud. 2017;75:35-42. doi:10.1016/j.ijnurstu.2017.06.014.

33. Xakellis GC, Frantz RA. The cost-effectiveness of interventions for preventing pressure ulcers. The Journal of the American Board of Family Practice / American Board of Family Practice. 1996;9(2):7985.

34. Xakellis GC, Jr., Frantz RA, Lewis A, Harvey P. Cost-effectiveness of an intensive pressure ulcer prevention protocol in long-term care. Adv Wound Care. 1998;11(1):22-9.

35. Bosanquet N, Franks P, Moffatt C, Connolly M, Oldroyd M, Brown P et al. Community leg ulcer clinics: cost-effectiveness. Health trends. 1993;25(4):146-8.

36. Korn P, Patel ST, Heller JA, Deitch JS, Krishnasastry KV, Bush HL et al. Why insurers should reimburse for compression stockings in patients with chronic venous stasis. Journal of Vascular Surgery. 2002;35(5):950-7. doi:10.1067/mva.2002.121984.

37. McGuckin M, Waterman R, Brooks J, Cherry G, Porten L, Hurley S et al. Validation of venous leg ulcer guidelines in the United States and United Kingdom. American Journal of Surgery.

2002;183(2):132-7. doi:10.1016/S0002-9610(01)00856-X. 
38. Simon DA, Freak L, Kinsella A, Walsh J, Lane D, Groarke L et al. Community leg ulcer clinics: a comparative study in two health authorities. Bmj. 1996;312:1648-51.

39. DaVanzo JE, El-Gamil AM, Dobson A, Sen N. A retrospective comparison of clinical outcomes and Medicare expenditures in skilled nursing facility residents with chronic wounds. Ostomy/Wound Management. 2010;56(9):44-54.

40. Graves N, Finlayson K, Gibb M, O'Reilly M, Edwards H. Modelling the economic benefits of gold standard care for chronic wounds in a community setting. Wound Practice and Research. 2014;22(3). 41. Harris C, Shannon R. An innovative enterostomal therapy nurse model of community wound care delivery: A retrospective cost-effectiveness analysis. Journal of Wound, Ostomy and Continence Nursing. 2008;35(2):169-83. doi:10.1097/01.WON.0000313639.37247.c0.

42. Rybak Z, Franks PJ, Krasowski G, Kalemba J, Glinka M. Strategy for the treatment of chronic leg wounds: A new model in Poland. International Angiology. 2012;31(6):550-6.

43. Vu T, Harris A, Duncan G, Sussman G. Cost-effectiveness of multidisciplinary wound care in nursing homes: A pseudo-randomized pragmatic cluster trial. Family Practice. 2007;24(4):372-9. doi:10.1093/fampra/cmm024.

44. International Working Group on the Diabetic Foot. International Consensus on the Diabetic Foot. Amsterdam, The Netherlands: International Working Group on the Diabetic Foot; 1999.

45. Haji Ali Afzali H, Gray J, Karnon J. Model Performance Evaluation (Validation and Calibration) in Model-based Studies of Therapeutic Interventions for Cardiovascular Diseases. Applied health economics and health policy. 2013;11(2):85-93. doi:10.1007/s40258-013-0012-6. 